\title{
DTX mechanism at Evolve Node for Efficient Power Saving in LTE Network
}

\author{
Vaibhav Gaikwad ${ }^{\mathrm{a}}$, Manish Sharma ${ }^{\mathrm{b}}$ and Trupti $\mathrm{Wagh}^{\mathrm{c}}$ \\ ${ }^{a}$ Dept. of Electronics and Telecommunication, D. Y. Patil College of Engineering, Pune-411044, India \\ ${ }^{b}$ Prof, Dept. of Electronics and Telecommunication, D. Y. Patil College of Engineering, Pune-411044, India \\ ${ }^{c}$ Asst. Prof, Dept. of Electronics and Telecommunication, D. Y. Patil College of Engineering, Pune-411044, \\ India
}

\begin{abstract}
Power in any cellular network is a key degree of freedom in management of connectivity, energy factors, speed etc. In LTE network the power consumption is dominated by Evolve Node by significant amount of power, to compute this issue a mechanism has been proposed to reduce the power consumption. The power consumption issue is tackle by Discontinuous Transmission (DTX) mechanism where the evolve node is switch off for specified amount of time when there is no occurrence of packet. These are simulated with busty traffic condition which is considered to poison traffic with exponential distribution for delay sensitive and insensitive traffic, where power consumption and latency average is calibrated.
\end{abstract}

Index Terms: LTE, DTX, Power Saving.

(C) 2015 Published by MECS Publisher. Selection and/or peer review under responsibility of the Research Association of Modern Education and Computer Science

\section{Introduction}

Long Term Evaluation (LTE), a new-generation technology for mobile communication was specially created to fulfil the need of bandwidth ravenous users. With aims to entitle users with a new smartphone experience, providing higher data-rates and lower latencies that could transform the overall industry into a new wireless ecosystem of smartphone devices and applications. LTE render remarkable improvements over older cellular communication standards like GPRS, EDGE and WCDMA, and because of this outstanding improvement it is called as 4G (fourth generation) technology. LTE architecture is based on Internet Protocol (IP) distinct from other cellular Internet protocols, the 4G-LTE supports browsing Web sites, VoIP and other IP-based services as well. LTE was proposed to achieve theoretically download rate of 300Mbps or more based on experimental trials. In practical the rates vary on actual network bandwidth accessible to an individual LTE subscriber

* Corresponding author

E-mail address: vsgaikwad02@gmail.com 
sharing the service provider's network (ISP) with other customers is significantly less [1]. An Evolve Node consume approximately $2 \mathrm{KW}$ of power, taking this in account the power consumption of User Equipment is negligible. So the total LTE network power utilization is currently dominated by Base band Station i.e Evolve Node. To tackle this power consumption problem different initiative are undertaken by big players of Communication market [1]. Project like 'Green Touch' and 'EARTH'. Another initiative was taken based on distance of UE to switch evolve Node B called GREEN DISTANCE AWARE eNB [2], where ON/OFF of trans-receiver is exploit depending on distance of User equipment from base station. In [5], an analysis was done based on an empirical power model for LTE system and they commented that LTE consume higher power than $3 \mathrm{G}$ and $\mathrm{WiFi}$ and it is 23 times less efficient. Even in EARTH project [3] power optimization is solved by SON (Self Optimization network) where a solution is provide to switch of relay of cell without disturbing Quality of service. In [4] a sophisticated analysis is done to measure the power consumption with different frequency considering Quality of service by optimizing modulation and coding schemes. [6][7] A Discontinuous transmission solution was proposed for evolve Node B to have power saving and power consumption is calculated for busty traffic. In this paper [6] excessive emission of $\mathrm{CO} 2$ is also taken in accordance with respective power consumption. In [6] author applied Discontinuous transmission base station (eNB) to save upto $70 \%$ of its energy. But they completely ignore other factor which also contribute in power consumption is backhauling and other essential component at base station site. In [7] power saving for user equipment has given importance as battery constraint of equipment is limited where traffic is consider poison traffic.

So the simplest solution to power consumption problem can be considered as Discontinuous transmission.

We divide this paper in three main sections $2^{\text {nd }}$ LTE Architecture, $3^{\text {rd }}$ Mechanism, $4^{\text {th }}$ Simulation and Conclusion.

\section{LTE Architecture}

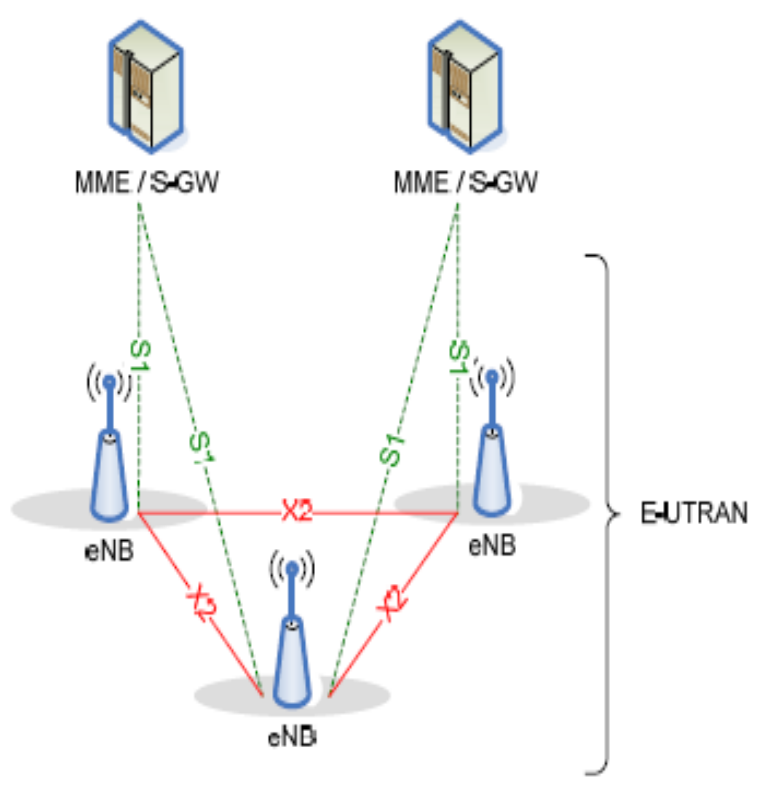

Fig.1. LTE UTRAN Architecture

The Long Term Evolution (LTE) system architecture is considered as shown in figure 1. 
LTE based on the legacy of its previous generation that is it is based on UTRAN architecture. Figure 1 depicts the essential design of LTE. The radio network architecture projected by the 3GPP LTE consists of evolved NodeB (eNodeB). The eNodeB provides a link between the user instrumentality and core network. As shown, eNodeB is connected to the core network via the $\mathrm{S} 1$ interface, and every eNodeB is interconnected via the $\mathrm{X} 2$ interface. The eNodeB is responsible for the bulk of the radio resource management (RRM) functions like packet programing. Both mobility management entity (MME) and serving gateway (S-GW) are a part of the core networks. The MME is responsible for paging and user instrumentality (UE/UI) quality in idle mode inside the network, whereas the S-GW node is responsible for routing user information packets and handling alternative user requests, as an example handover.

LTE uses orthogonal frequency division multiple access (OFDMA) as a radio interface. OFDMA divides the bandwidth into subcarriers and assigns them to the users depending on the present demand of service. Every subcarrier carries information at low rate, however exploitation of multiple subcarriers at once to produce high information rates.

\section{Proposed Mechanism: Discontinuous Transmission (DTX)}

As the overall LTE network power consumption is dominated by Base band station i.e eNB. So firstly area will be considered where the power is consumed at eNB site. In typical situation power is utilized by eNB base band part where the transmission, translation and connection are done. Secondly 'cooling', a significant power is consume to cool down the system and thirdly 'Lights'. In all the three part cooling is essential while lights can be turns off as per requirement, So only Base Band part remain which will be our main concern. Here we have considered the backhauling power ideally zero. Total power consume can be represented as

$$
P_{\text {total }}=P_{\text {baseband }}+P_{\text {cooling }}+P_{\text {light }}+P_{\text {backhauling }}
$$

So the $\mathrm{P}_{\text {total }}$ which is estimated to be $2 \mathrm{KW}$ in [6]. Our concern is $\mathrm{P}_{\text {baseband }}$ so to have effiecient power saving in $\mathrm{P}_{\text {baseband }}$ we apply discontinues transmission.

\subsection{Discontinues Transmission (DTX)}

LTE DTX has two states, DTX state and Idle state. At DTX state, the evolve node can be in one of the two modes: Continuous Transmission, Short DTX. The transition from one state to another depends on the traffic activity. Network runs an inactivity timer called timeout to push the device to Idle mode. When there is no packet activity for a long duration, evolve node is moved to Idle state from Connected state. After some time timeout expire to wake evolve node (eNB) is set with any uplink or downlink data activity. In LTE, eNB may be configured with a DTX mechanism in both Connected and Idle states. However, the functionality and configuration parameters of DTX are different in these LTE states. Since data traffic mainly take place in connected state. DTX can be configured on mainly data activity. Network runs a wakeup timer called DTX Timeout timer for the connected eNBs to push them from DTX mode. When there is no packet activity for certain duration the eNB's goes to sleep and if there is data activity then eNBs stays in connected state but moves to DTX mode. DTX timeout timer is also reset with any uplink or downlink data activity. Timeout timer is used to move the eNBs from Idle used to move the eNBs into DTX mode while still staying in Connected. In current LTE deployments, sleep time should larger than activity time to have large amount of power saving. 


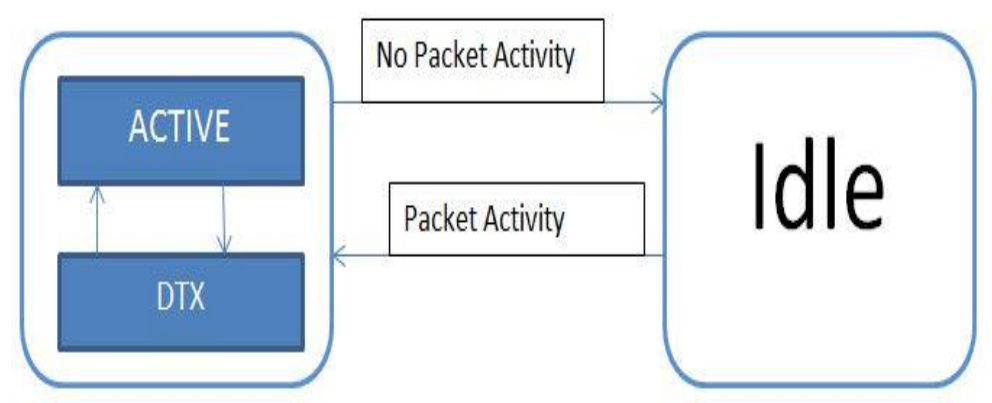

Fig.2. LTE DTX mode

Total baseband Power can represent by

$$
P_{\text {baseband }}=P_{R F}+P_{R C}
$$

where $P_{R F}$ power required by multi user software define radio (SDR) and $P_{R C}$ is actual physical component namely power amplifier, oscillator etc. in $\mathrm{P}_{\mathrm{RC}}$ actual power allocation in done, so $\mathrm{P}_{\mathrm{RC}}$ can be stated as

$$
P_{R C}=\mathrm{P}_{\mathrm{r} r}+\frac{P_{\text {carrier }}}{k} \times N_{\mathrm{sec}}
$$

Where $\mathrm{P}_{\mathrm{rr}}$ is constant power requires by the physical component, $\mathrm{P}_{\text {carrier }}$ is power allocation per symbol, $\mathrm{N}_{\mathrm{sec}}$ is number of sector and $\mathrm{k}$ is efficient factor.

\subsection{Modelling: Two State Model}

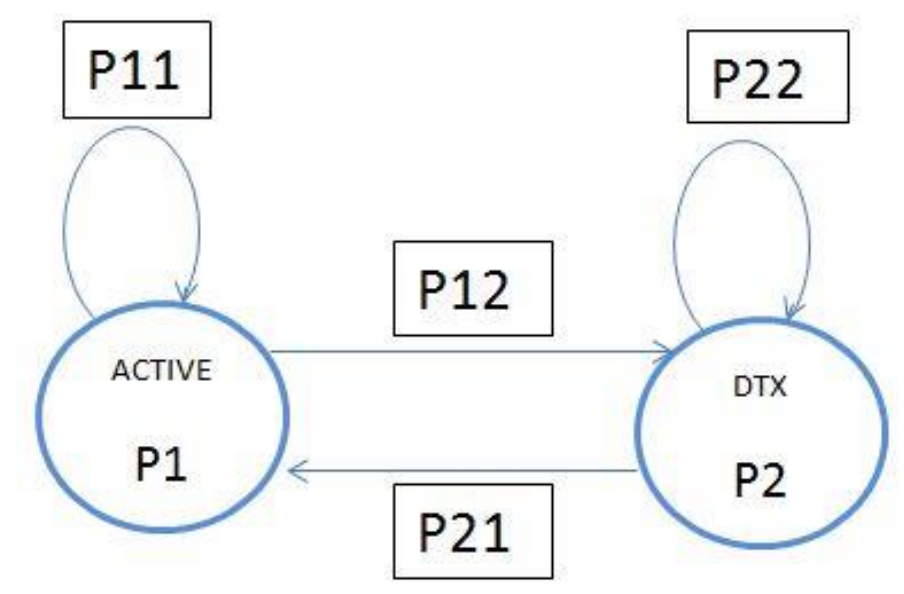

Fig.3. Two state Model 
Two state models is proposed where $\mathrm{P}_{1}$ active $\mathrm{P}_{2}$ is DTX mode with mirco sleep.

- State $\mathrm{P}_{1}$ comprises a sequence of adjacent active time intervals corresponding to the entire duration of a single packet call transmission, that is the eNB is in power active mode.

- State $\mathrm{P}_{2}$ comprises a sleep period (tdtx) that is entered from $\mathrm{P}_{1}$, that is the eNB follows DTX mirco cycles.

When there is on-going traffic condition the evolve node remain in active state as soon as evolve node detect that there is no occurrence of packet based on inter arrival time, it switch off some part of trans-receiver where as some part remain active to detect occurrence activity.

The evolve node revive from switch off mode to Active mode after a predefine timer which is timeout timer which trigger the evolve node to wake up and force it in active state. There is also second condition to wake up the evolve node by sending acknowledgement of emergency call to evolve node. The transition matrix can be given by

$$
\begin{aligned}
& y=\left(\begin{array}{cc}
P_{11} & P_{12} \\
1 & 0
\end{array}\right) \\
& \text { timer }_{\text {timeout }}=\frac{8 \times \text { Framelength }}{B W \times \mu} \\
& \text { wakeup }_{\text {delay }}=\sum_{i=0}^{\infty} \frac{T T I \times T_{d}}{2}
\end{aligned}
$$

Where timer ${ }_{\text {timeout }}$ is wakeup timer called timeout timer, BW is bandwidth and $\mu$ is efficiency factor, TTI inter arrival time and $\mathrm{T}_{\mathrm{d}}$ is time delay.

\section{Simulation and Results}

The simulation is perform in MATLAB with some predefine parameter's [5].

The above parameters are kept constant for simplicity of coding and we also assume than the transient loss are negligible which is loss of power moving from idle state to connected state.

Table 1. Parameter for Simulation

\begin{tabular}{|l|l|l|}
\hline Sr no. & Parameters & Values \\
\hline 1 & DTX sleep period & $30 \mu \mathrm{s}$ \\
\hline 2 & $\mathrm{P}_{\text {baseband }}$ & $200 \mathrm{~W}$ \\
\hline 3 & $\mathrm{P}_{\text {DTX }}$ power consume in DTX & $10 \mathrm{~W}$ \\
\hline 4 & $\mu$ & $20 \%$ \\
\hline 5 & $\mathrm{P}_{\text {carrier }}$ & $20 \mathrm{~W}$ \\
\hline 6 & Traffic & Poisson traffic for busty data with exponential distribution \\
\hline 7 & eNB & 40 considering Urban area with macro implementation \\
\hline
\end{tabular}

We calculated average power consumption and latency by two methods one is arithmetic average (AA) and another is the adaptive exponentially smoothed average (AeSA). Both the condition where taking in consideration such as busty traffic that is poison traffic with taking in account Quality of service such Delay sensitive (DST) traffic and Delay Insensitive Traffic (DIST). 
The Average power is calculated by

$$
\begin{aligned}
& \text { AvgPower }=\frac{P_{D T X}}{\mathrm{P}_{R C}} \times I T T \\
& \text { Power }_{\text {Idle }}=P_{R F}
\end{aligned}
$$

where ITT is number of inter arrival of number of packets.

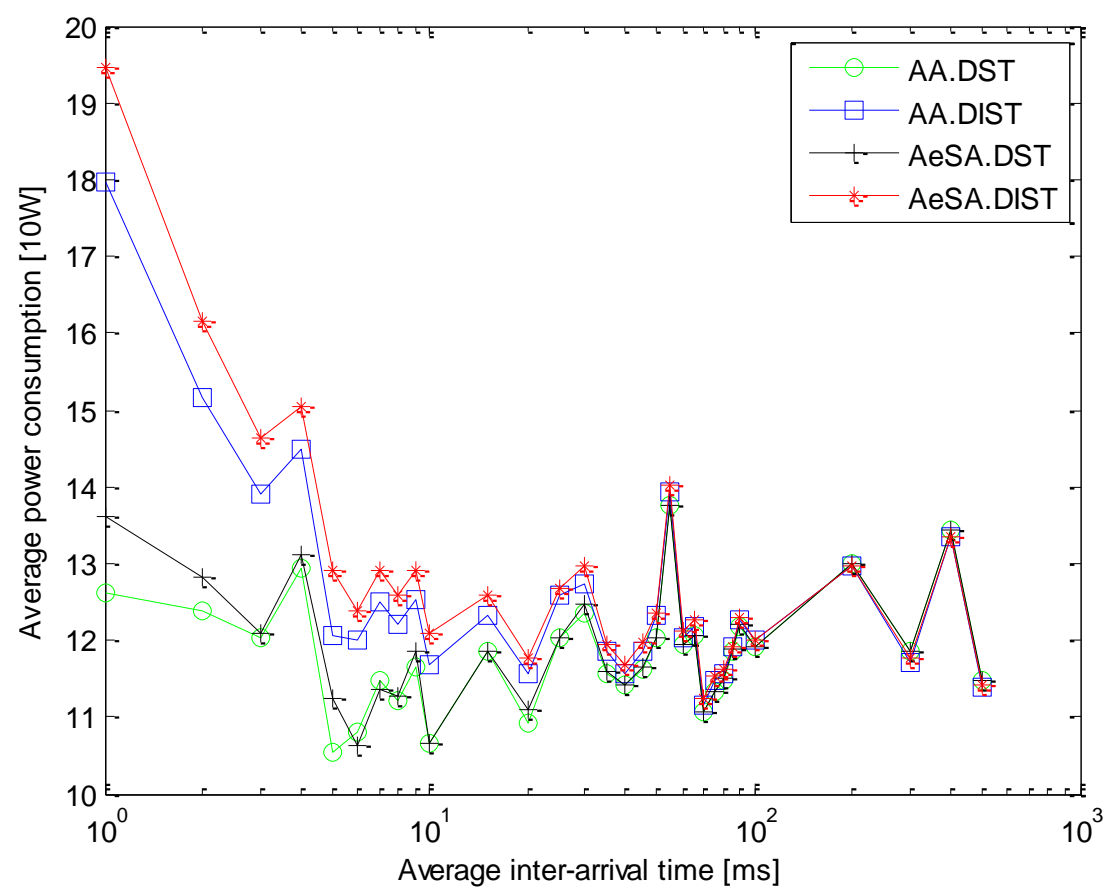

Fig.4. Power Consumption as Function of Inter Arrival Time

Figure 4 shows average power consumption with respect to average inter arrival time where evaluation is done using delay insensitive traffic as well as delay sensitive traffic where inter arrival time in both case are calibrated by two methods one is arithmetic average (AA) and another is the adaptive exponentially smoothed average (AeSA). Both the case that are delay insensitive traffic as well as delay sensitive traffic the power consumption is decreasing with increase in inter arrival time.

Figure 5 shows system performance where latency is shown as function of inter arrival time where latency is increasing as inter arrival time increase for both the case that are delay insensitive traffic as well as delay sensitive traffic. So there is need for proper trade-off between latency and power consumption.

Figure 6 shows average sleep ratio with respect to inter arrival time where sleep ratio is increasing with increasing in inter arrival time for both the cases delay insensitive traffic as well as delay sensitive traffic. 


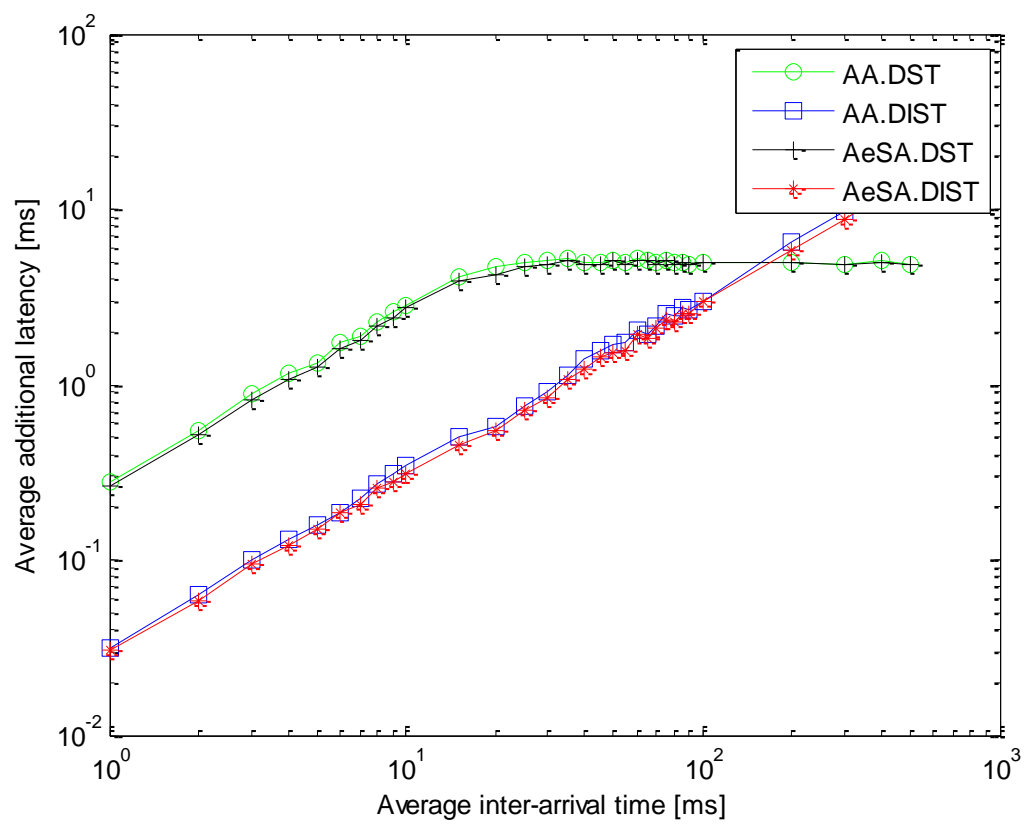

Fig.5. Latency as Function Inter Arrival Time

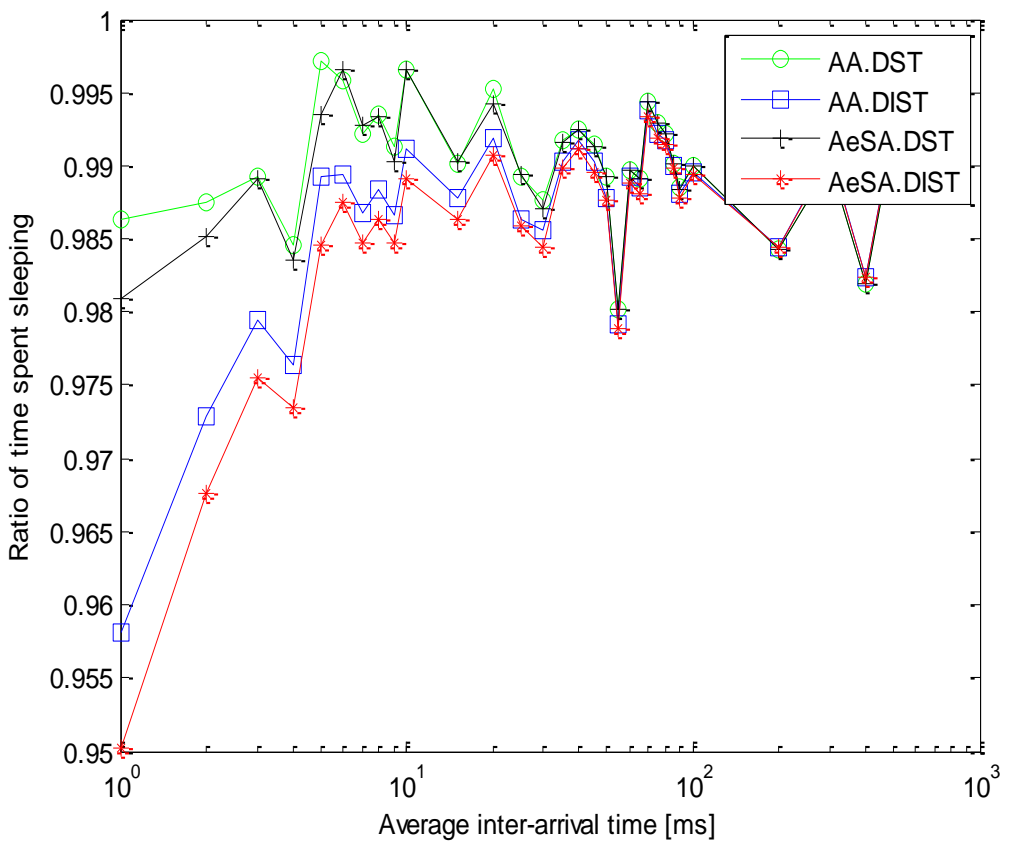

Fig.6. Sleeping time v/s IAT 
While performing DTX, a proper balance should be made between energy saving and latency because saving energy can drastically increase the latency which is not expected from LTE.

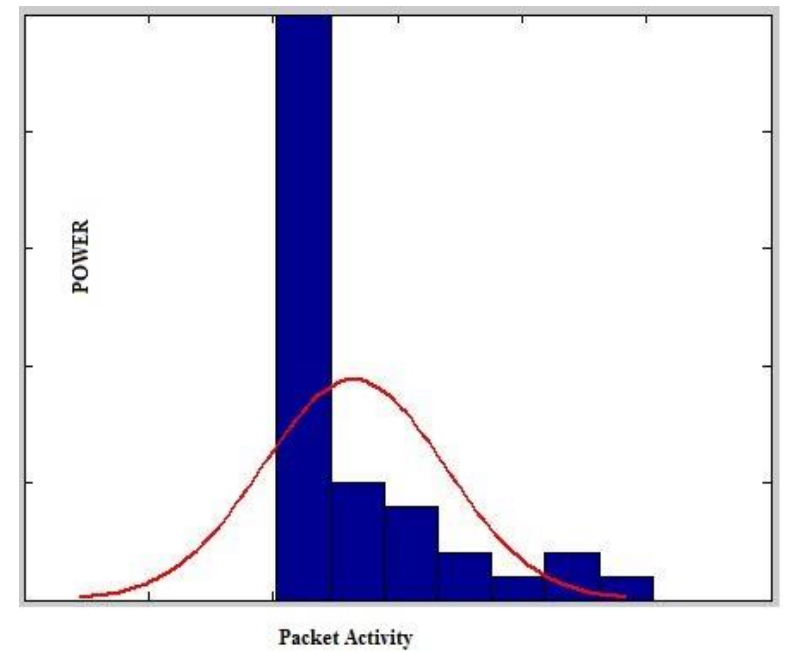

Fig.7. Power as Function of Packet Activity

\section{Conclusion}

An LTE is one of the most popular technology among user because of improved system capacity and coverage, High peak data rates, Low latency, and Multi-antenna support. But the problem which LTE suffers is from high power utilization which can be overcome by DTX in significant amount. A DTX is applied to a LTE network which show that the DTX can impact greatly on power consumption. By DTX of short $60 \mu$ s an power saving of almost $50 \%$ to $60 \%$ can be achieve in $\mathrm{P}_{\text {baseband }}$. But while consider base station site power DTX contribute $5 \%$ to $20 \%$ in real world.

\section{Acknowledgement}

I would like specially thank to Head of Department Prof.(Dr) D G Khairnar for his whole hearted cooperation, suggestion and technical guidance throughout the work.

\section{References}

[1] Xiaofei Wang, Athanasios V Vasilakos Min Chen Yunhao Liu Ted Taekyoung Kwon, "A Survey of Green Mobile Networks: Opportunities and Challenges," Mobile Network Application: Springer, vol. 17, no. 1, pp. 4- 20, Arpil. 2012.

[2] Mads Lauridsen, Anders Riis Jensen, and Preben Mogensen, Luis Alonso, and Christos Verikoukis, "Green" Distance-Aware Base Station Sleeping Algorithm in LTE-Advanced," IEEE Precedings on Communications (ICC), pp. 1347- 1351, Jun. 2012.

[3] Oliver Blume, Harald Eckhardt, Siegfried Klein, Edgar Kuehn, and Wieslawa M. Wajda, "Energy Savings in Mobile Networks Based on Adaptation to Traffic Statistics," Wiley Periodicals, Inc: Bell Labs Technical Journal,no.2 pp. 77-94, Jun. 2010. 
[4] Bjoern Dusza, Christoph Ide and Christian Wietfeld, "Measuring the Impact of the Mobile Radio Channel on the Energy Efficiency of LTE User Equipment," 21st International Conference on Computer Communication Networks, pp. 1- 5, 2012.

[5] Junxian Huang Feng Qian Alexandre Gerber Z. Morley Mao1 Subhabrata Sen Oliver Spatscheck, "A Close Examination of Performance and Power Characteristics of 4G LTE Networks," ACM: MobiSys'12, PP. 25-29, 2012.

[6] Pål Frenger, Peter Moberg, Jens Malmodin, Ylva Jading, and István Gódor, et al "Reducing Energy Consumption in LTE with Cell DTX," Proceedings IEEE VTS Vehicular Technology Conference., pp. 1- 5, May. 2011.

[7] S.-R. Yang, S.-Y. Yan, and H.-N. Hung, "Modeling UMTS power saving with bursty packet data traffic," IEEE Transaction Mobile Computing, vol. 6, no. 12, pp. 1398-1409, Dec. 2007.

[8] Gürkan Gür and Fatih Alagöz, "Green Wireless Communications via Cognitive Dimension: An Overview," IEEE Network, vol. 22, no. 2, pp. 50- 56, Mar. 2011.

[9] Alexandra Bousia, Angelos Antonopoulos, Luis Alonso, and Christos Verikoukis, "Reducing LTE Uplink Transmission Energy by Allocating Resources," Proceedings IEEE VTS Vehicular Technology Conference., pp. 1- 5, Sept. 2011.

[10] Vincenzo Mancuso a, Sara Alouf b, "Analysis of power saving with continuous connectivity," ELSEVIER: Computer Networks, Vol. 56 pp. 2481-2493, 2012.

[11] Chunyi Peng, Suk-Bok Lee, Songwu Lu, Haiyun Lu, Hewu Li, "Traffic-Driven Power Saving in Operational 3G Cellular Networks," ACM :MobiCom'11, PP. 19-23, 2011.

[12] Vaibhav Gaikwad and Trupti Wagh, "Overview of Power Optimization in LTE Network" IJCA Proceedings on National Conference on Emerging Trends in Advanced Communication Technologies NCETACT 2015, vol 5, pp 13-15, June 2015.

[13] Vaibhav Gaikwad, Manish Sharma, Trupti Wagh, "DRX and DTX Algorithms at BS and UE for Minimum Power Consumption in LTE Network." Journal of Advancements in Robotics, vol 2, issue 2, 26-32p. 2015.

[14] Ali T. Koc, Member, IEEE, Satish C. Jha, Member, IEEE, Rath Vannithamby, Senior Member, IEEE, and Murat Torlak, Senior Member, IEEE, "Device Power Saving and Latency Optimization in LTE-A Networks Through DRX Configuration," IEEE Transaction Wireless Communications, vol. 13, no. 5, MAY 2014.

[15] Ayman Elnashar and Mohamed A. El-Saidny, "Extending the Battery Life of Smartphones and Tablets," IEEE vehicular technology magazine JUNE 2014.

\section{Author(s) Profiles}

Vaibhav Gaikwad pursing Master's in Communication Network from D. Y. Patil College of Engineering, Akrudi, Pune in University of Pune.

Dr. Manish Sharma Professor in D. Y. Patil College of Engineering, Akurdi, Pune.

Trupti Wagh Assistant Professor in D. Y. Patil College of Engineering, Akrudi, Pune. 